Group field theory and simplicial quantum gravity

This article has been downloaded from IOPscience. Please scroll down to see the full text article.

2010 Class. Quantum Grav. 27145017

(http://iopscience.iop.org/0264-9381/27/14/145017)

View the table of contents for this issue, or go to the journal homepage for more

Download details:

IP Address: 194.94.224.254

The article was downloaded on 02/04/2013 at 12:19

Please note that terms and conditions apply. 


\title{
Group field theory and simplicial quantum gravity
}

\author{
D Oriti \\ Institute for Theoretical Physics, Utrecht University, Leuvenlaan 4, Utrecht 3584 TD, \\ The Netherlands \\ Perimeter Institute for Theoretical Physics, 31 Caroline St, Waterloo, Ontario N2 L 2Y5, Canada \\ and \\ Albert Einstein Institute, Am Muehlenberg 4, Golm, Germany \\ E-mail: doriti@perimeterinstitute.ca, daniele.oriti@aei.mpg.de
}

Received 29 October 2009, in final form 22 April 2010

Published 8 June 2010

Online at stacks.iop.org/CQG/27/145017

\begin{abstract}
We present a new group field theory for 4D quantum gravity. It incorporates the constraints that give gravity from BF theory and has quantum amplitudes with the explicit form of simplicial path integrals for first-order gravity. The geometric interpretation of the variables and of the contributions to the quantum amplitudes is manifest. This allows a direct link with other simplicial gravity approaches, like quantum Regge calculus, in the form of the amplitudes of the model, and dynamical triangulations, which we show to correspond to a simple restriction of the same.
\end{abstract}

PACS numbers: $04.60 . \mathrm{Pp}, 04.60 . \mathrm{Nc}, 04.60 . \mathrm{Gw}$

\section{Introduction and motivation}

\subsection{GFTs, spin foams and simplicial gravity}

The field of non-perturbative quantum gravity is progressing fast [1] in several directions. Spin foam models [10] are one of them and can be understood as a covariant formulation of the dynamics of loop quantum gravity [11] and as a new algebraic version of the discrete quantum gravity approach based on path integrals, as for example Regge calculus [13] and dynamical triangulations [14]. This line of research has recently received further impetus with the introduction of new models for four-dimensional quantum gravity in [22, 24-26]. The general idea of spin foam models is to encode the kinematics of quantum gravity in discrete quantum histories given by spin foams: combinatorial 2-complexes labeled by group-theoretic data. The 2-complex is combinatorially dual to a simplicial complex, and the algebraic data are interpreted as determining a possible simplicial geometry, just as edge lengths do in traditional Regge calculus. A quantum dynamics is specified by the assignment of a probability amplitude to each spin foam, with the model being defined by a sum over both 2-complexes and algebraic 
data labeling them. At present the most complete definition of a spin foam model is achieved by means of the so-called group field theory formalism [2-4]. Group field theories are quantum field theories over group manifolds (usually the Lorentz group) characterized by a non-local pairing of field arguments in the action and can be seen as a generalization of matrix models [5] that have proven so useful for our understanding of 2D quantum gravity (and string theory) (and of the subsequent, but less developed, tensor models [6, 7]). The combinatorics of the field arguments in the interaction term of the group field theory (GFT) action matches the combinatorics of $(d-2)$ faces of a $d$-simplex, with the GFT field itself interpreted as a (second) quantization of a $(d-1)$-simplex. The kinetic term of the action, in turn, dictates the rules for gluing two such $d$-simplices across a common $(d-1)$-simplex, and thus for the propagation of (pre-)geometric degrees of freedom from one to the next. See [2,3] for details. Because of this combinatorial structure, the Feynman diagrams of a GFT are dual to $d$-dimensional simplicial complexes and are themselves given by 2 -complexes. The field arguments assign to these 2-complexes the same group-theoretic data that characterize spin foam models, and, most importantly, the GFT perturbative expansion in Feynman amplitudes defines uniquely and completely [8] a spin foam model. This fact alone makes GFTs a very useful tool, and also leads to the suggestion that they can provide a more fundamental definition of a dynamical theory of spin networks (as a second quantized theory of spin networks, clarified also in [12]), in particular representing the best way to investigate non-perturbative and collective properties of their quantum dynamics [9]. It can also be argued [2, 3, 9] that GFTs represent a common framework for both the loop quantum gravity/spin foam approach and simplicial approaches, like quantum Regge calculus and (causal) dynamical triangulations, whose basic ideas and structures they incorporate.

\subsection{Motivation}

For this idea to be realized, or at least investigated in more concrete terms, it is necessary to have at one's disposal a class of group field theories whose Feynman (spin foam) amplitudes are given by path integrals for simplicial gravity actions in the first-order form (as appropriate for the type of variables appearing in them). This has been partially achieved in [16]. In this paper, we develop further, and in a sense bring to a completion, the work in [16] and present a GFT for four-dimensional quantum gravity, whose Feynman amplitudes are indeed simplicial gravity path integrals with a clearly identified simplicial action and with all the GFT variables possessing a clear geometric interpretation. More precisely, the model's amplitudes are path integrals for a discrete Plebanski action, i.e. a discrete BF action with constraints on the $B$ variables that turn them into a discretization of the continuum tetrad field [10], augmented by quantum corrections. On the one hand, therefore, we obtain an explicit link with 4D simplicial quantum gravity and with other simplicial approaches. On the other hand, the ability to control the imposition of the constraints in a path integral context makes the proposed GFT a good step forward toward a spin foam formulation of 4D quantum gravity, since the correct imposition of these constraints is the declared goal of all current spin foam models [22, 24-26] (we will discuss in the following both the good sides and the limitations of the model we are presenting). We achieve this by means of a generalization of the whole GFT formalism that allows us to bypass some not entirely satisfactory (in our opinion) steps in the usual spin foam quantization procedure, when re-phrased in a Lagrangian path integral language. Let us now sketch briefly these steps and the reasons why we find them problematic.

The classical inspiration is the Plebanski formulation of gravity as constrained BF theory [10]. This means that the theory one would really want to quantize in the path integral 
(Lagrangian) language is some discrete version of the Plebanski action on a simplicial complex $\Delta$, schematically of the form

$$
Z_{\Delta}=\int \mathcal{D} g_{e} \mathcal{D} B_{f} \prod_{e} C\left(B_{f \subset e}\right) \mathrm{e}^{\mathrm{i} \sum_{f} \operatorname{tr}\left(B_{f} H_{f}\left(g_{e \in \partial f}\right)\right)} .
$$

Here the starting point at the classical level is the action for BF theory discretized on the simplicial complex. Lie algebra variables $B_{f} \in \mathfrak{s o}(4)$ are associated with all the triangles $f$ of the complex; group elements $g_{e}$ are associated with the tetrahedra $e$ of the complex, and represent parallel transports of a discrete connection from a given 4-simplex to a neighboring one, across the given tetrahedron $e$ shared by the two 4-simplices. Both sets of variables are of course integrated over in the formal definition (before gauge fixing) of the partition function of the theory. The curvature associated again with each triangle is obtained from the elementary connection variables as $H_{f}=\prod_{e \in \partial f} g_{e}$, where the product runs over the tetrahedra $e$ sharing the triangle $f$. The trace is in the fundamental representation. This defines the discrete BF action. Then, we have simplicity constraints at the level of each tetrahedron of the simplicial complex [22] (see also [29]). These constraints impose the restriction on the classical discrete Lie algebra $B$ variables constraining the set of $B_{f}$ variables to be determined by a discrete tetrad field [28] and thus giving gravity from BF theory. We will give the precise form of these constraints in the following. Secondary constraints arise, in the continuum, from commutators of simplicity constraints with the other constraints of the theory [32]. Similarly, additional (secondary) constraints, on top of the simplicity constraints, have been argued to be necessary also in a discrete context [30, 38]. For example, in the discrete context one associated reference frames with the different tetrahedra in the simplicial complex, and then imposes simplicity constraints separately in each tetrahedron. One then expects additional constraints enforcing the correct parallel transport of such constraints from one frame to another. Any such additional constraint is to be understood as a constraint on the discrete connection variables [30, 32, 38], allowing us in particular to determine the intrinsic (spatial) part of the connection degrees of freedom to the tetrad or Lie algebra $B$ variables, while the remaining degrees of freedom would define the extrinsic curvature. It is not clear, at present, if and how such additional constraints are accounted for in recent spin foam models. We will see how similar restrictions on the connection, possibly related to the ones identified in $[30,38]$, arise naturally in our model.

The usual spin foam procedure [10] mimics this path integral quantization but is applied at the level of quantum states directly, using methods from geometric quantization. The idea is as follows. We do know the structure of states of quantum BF theory formulated as a spin foam model, the Ooguri-Crane-Yetter model (being the 4D counterpart of the Ponzano-Regge model for 3D gravity/BF), adapted to a simplicial complex: they are given by spin networks with links labeled by representations of $S O(4)$ (in Euclidean signature), with 4-valent vertices dual to tetrahedra of the corresponding simplicial complex (while their links are dual to the triangles of the same tetrahedra). We also know how to construct, starting from such states, an amplitude for a single 4-simplex that is then a function of the representations labeling its ten triangles and the additional parameters labeling its five boundary tetrahedra. It is obtained by appropriate contraction of the five intertwining tensors associated with its five boundary tetrahedra. This is the main ingredient of the spin foam model defining the dynamics of the BF quantum states. The other contributions to the complete spin foam amplitude, associated with lower-dimensional simplices, i.e. triangles and tetrahedra, and usually thought of as 'measure terms' can similarly be defined, but can be determined uniquely only by the requirement of triangulation independence (following from the topological character of quantum BF theory). The task for defining a spin foam dynamics for $4 \mathrm{D}$ gravity is then first of all to find appropriate 
restrictions on the $S O(4)$ spin networks of BF theory, i.e. on their representation and intertwiner labels, that can be interpreted as the quantum analog of the Plebanski constraints in the classical $B$ variables of BF theory, and thus to characterize the spin network quantum states of $4 \mathrm{D}$ gravity. In the new spin foam models of [22, 24-26], the crucial intermediate step is to rewrite the same spin network states of BF theory in terms of coherent states of $S O(4)$, whose defining parameters are interpreted as the quantum labels corresponding to the $B$ variables. Second, one follows the steps leading to the BF spin foam amplitudes, but starting the newly defined candidate 4D gravity states, arriving at a proposal for the 4-simplex gravity amplitude. This procedure again leaves the measure terms in the spin foam model undetermined, and this time we do not expect, and thus we cannot impose, any triangulation independence property. Moreover, the identification of the analog of the discrete classical $B$ variables, e.g. with the parameters labeling the quantum coherent states, in the quantized BF partition function or in its quantum states expressed in terms of group elements or group representations is not free of ambiguities $^{1}$. Consequently, the procedure for imposing the simplicity constraints on them, that would be rather straightforward at the path integral level, is itself ambiguous.

When re-phrased in terms of a path integral quantization, the same spin foam procedure consists of four basic steps: (1) start from the BF path integral discretized on a simplicial complex, with the $B$ field discretized in terms of Lie algebra elements $B_{t}$ associated with the triangles of the complex, and the connection degrees of freedom encoded in group elements (to be thought of as parallel transports of the same connection) associated with the edges of the dual 2-complex and thus in 1-1 correspondence with the tetrahedra of the triangulation; (2) integrate out the $B_{t}$ variables so that one is left with a quantum amplitude function of group elements only, and given by a delta function for each triangle (dual face) imposing flatness of the corresponding holonomy (this is the entire content of BF theory); (3) expand the delta function in irreducible representations of the Lorentz group obtaining a re-formulation of the original discrete path integral in terms of group elements and representations of the same group; (4) identify in this reformulation variables that can be argued to correspond to the original Lie algebra variables $B_{t}$ and impose on them an analog of the Plebanski constraints on the $B$ field, modifying the measure on such variables in the partition function. This last step leaves us with a candidate partition function for 4D quantum gravity, that can then be put in a pure spin foam form by integrating out the group variables. In the most recent spin foam models [22, 24-26], for example, a basis of coherent states for the Lorentz group is used for expressing the amplitudes in the representation picture, i.e. after the expansion in step (3), their characterizing parameters are identified with the discrete $B$ variables, and the simplicity (Plebanski) constraints are imposed on them to give the final model. The initial BF path integral with group elements and Lie algebra variables does not play an essential role and one could have as well started directly from a definition of BF theory as a product of delta functions on the group, i.e. from the result of step $(2)^{2}$.

1 For example, as the parameters labeling the coherent states correspond to the mean value only of the operators corresponding to the $B$ variables, when computed in the same coherent states, one could argue that they can be identified with the same $B$ variables only in some semi-classical approximation, and not in the fundamental theory; consequently, the Plebanski constraints should not be imposed on these parameters, as done in the new spin foam models, strongly.

2 The model presented in [22] was recently given a simplicial path integral form in [27], used in [28] to study its semiclassical limit. The resulting simplicial action is a non-standard BF-like action involving explicitly discrete bivectors $B$ which are, however, defined in term of the group representations and coherent state parameters mentioned above, and not introduced as independent Lie algebra elements and later identified with the above in some approximation, as one would expect. So, the model can still be understood in the context outlined above, and thus subject to the same criticism. Concerning this last model as well as the problems in interpreting it in a path integral context, see [37]. 
The corresponding GFT construction of the same spin foam models follows an analogous path (see [10] and, for the new models, [22]). One starts from a GFT action designed to produce Feynman amplitudes given by products of delta functions. The action for such model (for $\phi \in \mathbb{R}$ ) is [15]

$$
\begin{aligned}
S[\phi]= & \frac{1}{2} \int \mathrm{d} g_{1} \ldots \mathrm{d} g_{4} \phi\left(g_{1}, g_{2}, g_{3}, g_{4}\right) \phi\left(g_{4}, g_{3}, g_{2}, g_{1}\right) \\
& -\frac{\lambda}{5 !} \int \mathrm{d} g_{1} \ldots \mathrm{d} g_{10}\left[\phi\left(g_{1}, g_{2}, g_{3}, g_{4}\right) \phi\left(g_{4}, g_{5}, g_{6}, g_{7}\right) \phi\left(g_{7}, g_{3}, g_{8}, g_{9}\right)\right. \\
& \left.\times \phi\left(g_{9}, g_{6}, g_{2}, g_{10}\right) \phi\left(g_{10}, g_{8}, g_{5}, g_{1}\right)\right]
\end{aligned}
$$

The field is assumed to be symmetric under the diagonal (right) action of the Lorentz group $G \phi\left(g_{i}\right)=\phi\left(g_{i} g\right) \quad \forall g \in G$. This symmetry requirement is ultimately responsible for the form of the quantum amplitudes. It introduces a gauge connection on the dual links of the simplicial complex and imposes flatness inside each 4-simplex, which, combined with the trivial gluing condition imposed by the kinetic term, results in imposing flatness on each dual face (triangle). Starting from the above, the crucial step is the insertion of appropriate constraints in the GFT action, in the above group picture (e.g. for the Barrett-Crane model [10]) or, in the representation picture, following steps (3) and (4) above. For example, in the new models [22], one can expand the GFT kinetic term in group representations, and then in coherent states of $G$, and finally constrain the corresponding parameters as the simplicial geometry of a tetrahedron (corresponding to the GFT field), when expressed in terms of bivectors $B_{t}$ suggests [26]. Once more, there is no role for the Lie algebra variables that would directly correspond to the discrete $B$ field.

There are reasons to be dissatisfied with this procedure, and to look for alternatives, in particular to look for a proper Lagrangian formulation of a 4D spin foam model for gravity.

We have already mentioned the difficulty in specifying uniquely the measure terms in the usual spin foam construction. A proper Lagrangian path integral derivation starting from $\mathrm{BF}$ theory (whose measure is unambiguous) could provide a prescription for these measure terms.

In a Lagrangian/path integral context, the two sets of Lie algebra and group variables are classical and independent of each other. Any relation between the two should arise in some (dominant) configurations, satisfying the discrete equations of motion. We want to reproduce exactly this feature, with a corresponding 'doubling' of independent geometric variables, at the GFT level, with our generalized GFT formalism. Alternatively, the relation between the two should be in terms of some classical Fourier transform mapping one set of variables to the other. We will discuss briefly this possibility in the following. On the other hand, the usual spin foam quantization introduces a quantum analog of the $B$ variables in terms of the Lie algebra generators acting on the function of the group variables, and thus lives from the start at the quantum (canonical) level, outside the path integral context.

Once an appropriate discretization of the simplicity constraints has been chosen, they are imposed as delta function restriction in the path integral measure on the classical independent $B$ variables. This means that, contrary to steps (3) and (4) above, there is no ambiguity in how to impose the simplicity constraints, which is the point of debate in the usual spin foam construction. Furthermore, this also means that they have to be imposed before performing the integration over these variables, i.e. at the classical level, as they drastically affect this integration, contrary to the usual spin foam procedure. 
In this work, we try to overcome the pitfalls of the usual procedure when seen from a Lagrangian path integral point of view ${ }^{3}$. We also make direct contact with simplicial gravity at the level of the resulting partition function (and not only as a guideline for the construction). We work in (and develop further) a generalized group field theory framework in which both group variables (representing discrete connection variables) and Lie algebra elements (representing discrete $B$ variables) are present. We obtain an explicit simplicial gravity path integral for 4D gravity in first-order variables, with BF-like action and the simplicity constraints manifestly implemented on the Lie algebra variables. Moreover, thanks to the presence of both sets of variables, we can keep under control and manifest the simplicial geometric picture at all stages in the construction. This also allows us to establish a direct link with other simplicial gravity approaches like quantum Regge calculus, in the very form of the quantum amplitudes of the model, and dynamical triangulations, which we can show to correspond to a simple restriction of the same.

The new model can be understood as a refinement of the class of models introduced in [16]. More details on the new 4D gravity model, together with the construction and thorough analysis of the simpler 3D gravity model, can instead be found in [21]. This work can also be understood in the context of the line of research on the issue of causality in spin foam quantum gravity and GFT, and on the construction of a unified GFT framework for loop quantum gravity and simplicial quantum gravity, developed in [16-18, 20].

\section{New GFT model for 4D quantum gravity}

\subsection{Definition of the model}

As it is usually the case in group field theory [2], our model, defining a theory in $d$ spacetime dimensions, is based on a (real) field $\phi\left(z_{1}, z_{2}, \ldots, z_{d}\right)$ of $d$ variables, interpreted as (pre-)geometric data, and by a classical action for this field of the general form:

$S(\phi)=\int \mathrm{d} z_{i} \phi\left(z_{i}\right)(\mathcal{K} \phi)\left(z_{i}\right)-\frac{\lambda}{d+1 !} \int \mathrm{d} z_{i j} \phi\left(z_{1 j}\right) \phi\left(z_{2 j}\right) \ldots \phi\left(z_{(d+1) j}\right) \mathcal{V}\left(z_{i j}\right)$

with a local quadratic term (the arguments of the two fields in it are identified) defined by some (differential) operator $\mathcal{K}$ and a combinatorially non-local interaction term, defined by the operator $\mathcal{V}$, which couples in a peculiarly non-local way the arguments of $d+1$ fields. The combinatorics of field arguments in the action, in particular in the interaction term, is best understood in terms of a diagram (representing the $d=4$ case) (see figure 1).

In the vertex term, in particular, each of the four arguments of each field is identified with one and only one argument of another field. Interpreting each field as a tetrahedron, with its four arguments corresponding to the four triangles in the boundary of the same tetrahedron, the above identification corresponds to the gluing of two tetrahedra along a common triangle, and the combinatorics of arguments in the GFT vertex is that of a 4-simplex [2] with five tetrahedra glued along triangles. The kinetic term, on the other hand, represents the gluing of two 4-simplices along a common tetrahedron obtained by identification of the corresponding boundary triangles. Within this interpretation, the indices $i$ in the kinetic term above identify the variables associated with the four triangles in the tetrahedron represented by the field, while, in the vertex term, the variables of the field are labeled by two indices $i j$ each corresponding to

\footnotetext{
3 Some of these motivations, and similar technical issues, are shared by the work [29] where a Lagrangian approach to a $4 \mathrm{D}$ gravity spin foam model on a given simplicial complex is presented. In the same way of reasoning, see the recent results of $[36,37]$, appeared after the completion of this work, and that we will discuss more in the following, as on the one hand they substantiate further the above criticisms, and on the other hand clarify better some of our own results.
} 


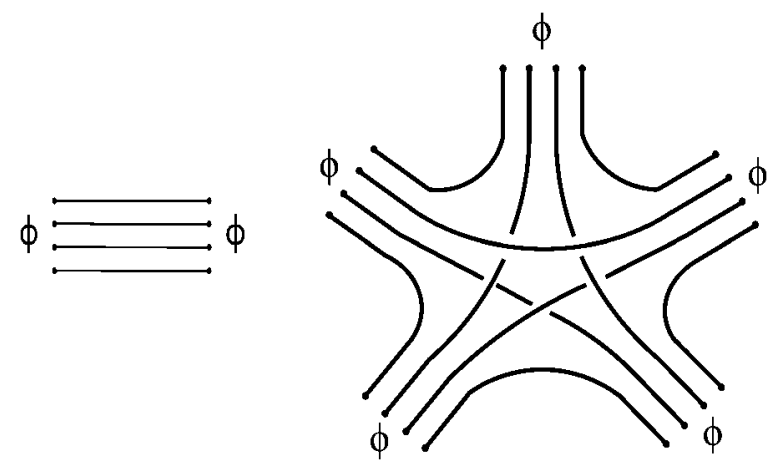

Figure 1. Kinetic and vertex term of 4D group field theory.

a tetrahedron in the 4-simplex, so that the pair of indices correspond to their common triangle, with the indices $i j$ and $j i$ associated with the same triangle seen in the two tetrahedra $i$ and $j$ respectively.

To be more specific, the field of our $\operatorname{model}^{4} \phi\left(x_{1}, b_{1}^{+} ; x_{2}, b_{2}^{+} ; x_{3}, b_{3}^{+} ; x_{4}, b_{4}^{+}\right)$lives on the Cartesian product of four copies of $S^{3} \times \mathfrak{s u}(2)$. For our present concerns (but see the discussion in [16]), we can restrict our attention to real fields. In turn, this field is obtained from a generic (real) field $\varphi\left(g_{1}, B_{1} ; \ldots ; g_{4}, B_{4}\right)=\varphi\left(g_{1},\left(b_{1}^{+}, b_{1}^{-}\right) ; \ldots ; g_{4},\left(b_{4}^{+}, b_{4}^{-}\right)\right)$on $(\operatorname{Spin}(4) \times \mathfrak{s o}(4))^{4}$ by application of the two maps $P_{B}$ and $P_{h}$, to be defined below: $\phi=P_{B} P_{h} \varphi$. In terms of these maps, as we are about to see in more details, one identifies the domain of dependence of $\phi, ' S^{3}$, , as the quotient of the domain of $\varphi$, 'Spin(4)', by the diagonal $S U(2)$ subgroup, and the $\mathfrak{s u}(2)$ algebra on which $\phi$ depends as the self-dual part of the Spin(4) algebra on which $\varphi$ depends.

The action defining the classical dynamics of the model is then given by

$$
\begin{aligned}
S= & \frac{1}{2} \int \mathcal{D} x_{i} \mathcal{D} b_{i}^{+} \phi\left(x_{i} ; b_{i}^{+}\right) \mathcal{K}_{m}\left(x_{i}, b_{i}^{+}\right) \phi\left(x_{i} ; b_{i}^{+}\right) \\
& -\frac{\lambda}{5 !} \int \mathcal{D} x_{i j} \mathcal{D} b_{i j}^{+}\left[P_{g} \phi\right]\left(x_{1 j} ; b_{1 j}^{+}\right) \ldots\left[P_{g} \phi\right]\left(x_{5 j} ; b_{5 j}^{+}\right) \mathcal{V}\left(x_{i j}, b_{i j}\right) \\
= & \frac{1}{2} \int \mathcal{D} x_{i} \mathcal{D} b_{i}^{+}\left[P_{B} P_{h} \varphi\right]\left(x_{i} ; b_{i}^{+}\right) \prod_{i}\left(\square_{i}^{S^{3}}+\left|b_{i}^{+}\right|^{2}-\frac{m^{2}}{4}\right)\left[P_{B} P_{h} \varphi\right]\left(x_{i} ; b_{i}^{+}\right) \\
& -\frac{\lambda}{5 !} \int \mathcal{D} x_{i j} \mathcal{D} b_{i j}^{+}\left[P_{g} P_{B} P_{h} \varphi\right]\left(x_{1 j} ; b_{1 j}^{+}\right) \ldots\left[P_{g} P_{B} P_{h} \varphi\right]\left(x_{5 j} ; b_{5 j}^{+}\right) \mathcal{V}\left(x_{i j}, b_{i j}\right) \\
= & \frac{1}{2} \int \mathcal{D} g_{i} \mathcal{D} \tilde{g}_{i} \int \mathcal{D} b_{i}^{+} \mathcal{D} b_{i}^{-} \int \mathcal{D} \tilde{b}_{i}^{+} \mathcal{D} \tilde{b}_{i}^{-} \int \mathcal{D} h_{i} \mathcal{D} \tilde{h}_{i} \int \mathcal{D} N \int \mathcal{D} \tilde{N} \\
& \times \prod_{i} \delta\left(b_{i}^{-}+N b_{i}^{+} N^{-1}\right) \delta\left(\sum_{i} b_{i}^{+}\right) \prod_{i} \delta\left(\tilde{b}_{i}^{-}+\tilde{N} \tilde{b}_{i}^{+} \tilde{N}^{-1}\right) \delta\left(\sum_{i} \tilde{b}_{i}^{+}\right) \\
& \times \varphi\left(g_{i} h_{i} ;\left(b_{i}^{+}, b_{i}^{-}\right)\right)\left[\prod_{i}\left(\square_{i}^{S^{3}}+\left|\tilde{b}_{i}^{+}\right|^{2}-\frac{m^{2}}{4}\right) \delta\left(g_{i} \tilde{g}_{i}^{-1}\right) \delta\left(b_{i}^{+}-\tilde{b}_{i}^{+}\right)\right] \varphi\left(\tilde{g}_{i} \tilde{h}_{i} ;\left(\tilde{b}_{i}^{+}, \tilde{b}_{i}^{-}\right)\right)
\end{aligned}
$$

4 We deal here with the Riemannian version of the new model. 


$$
\begin{aligned}
& -\frac{\lambda}{5 !} \int \mathcal{D} x_{i j} \mathcal{D} b_{i j}^{+}\left[P_{g} P_{B} P_{h} \varphi\right]\left(x_{1 j} ; b_{1 j}^{+}\right) \ldots\left[P_{g} P_{B} P_{h} \varphi\right]\left(x_{5 j} ; b_{5 j}^{+}\right) \\
= & \frac{1}{2} \int \mathcal{D} g_{i} \mathcal{D} \tilde{g}_{i} \int \mathcal{D} B_{i} \mathcal{D} \tilde{B}_{i} C\left(B_{i}\right) C\left(\tilde{B}_{i}\right) \varphi\left(g_{i} ; B_{i}\right) \\
& \times\left[\prod_{i} \int \mathcal{D} h_{i}\left(\square_{i}^{S p i n(4)}+\frac{1}{2}\left|\tilde{B}_{i}\right|^{2}-\frac{m^{2}}{4}\right) \delta\left(g_{i} h_{i} \tilde{g}_{i}^{-1}\right) \delta\left(b_{i}^{+}-\tilde{b}_{i}^{+}\right)\right] \varphi\left(\tilde{g}_{i} ; \tilde{B}_{i}\right) \\
& -\frac{\lambda}{5 !} \int \mathcal{D} x_{i j} \mathcal{D} b_{i j}^{+}\left[P_{g} P_{B} P_{h} \varphi\right]\left(x_{1 j} ; b_{1 j}^{+}\right) \ldots\left[P_{g} P_{B} P_{h} \varphi\right]\left(x_{5 j} ; b_{5 j}^{+}\right) \mathcal{V}\left(x_{i j}, b_{i j}\right)
\end{aligned}
$$

with the following ingredients.

- The measures of integration $\mathcal{D} g, \mathcal{D} h$ and $\mathcal{D} x$, as the standard Haar measures on Spin(4) and $S U(2)$ respectively, and the corresponding induced measure on the homogeneous space $S^{3} \simeq \operatorname{Spin}(4) / S U(2), \mathcal{D} B$ and $\mathcal{D} b$ are instead the standard Lebesgue measure on the Lie algebra considered as a vector space.

- The map $P_{B}$, acting on functions of $\mathfrak{s o}(4)^{4}$ as $P_{B} F\left(B_{1}, \ldots, B_{4}\right)=$ $P_{B} F\left(\left(b_{1}^{+}, b_{1}^{-}\right), \ldots,\left(b_{4}^{+}, b_{4}^{-}\right)\right)=\prod_{i} \int \mathcal{D} b_{i}^{-} \int_{S^{3}} \mathcal{D} N \int \mathcal{D} \tilde{N} \prod_{i} \delta\left(b_{i}^{-}+N b_{i}^{+} N^{-1}\right) \delta$ $\left(\sum_{i} b_{i}^{+}\right) F\left(\left(b_{1}^{+}, b_{1}^{-}\right), \ldots,\left(b_{4}^{+}, b_{4}^{-}\right)\right)=\prod_{i} \int \mathcal{D} b_{i}^{-} \mathcal{C}\left(B_{i}\right) F\left(B_{1} ; \ldots ; B_{4}\right)$ constrains the four Lie algebra variables associated with the field $\varphi$ imposing the simplicity constraints; its geometric meaning will be discussed below.

- The projector $P_{h}$ maps $\varphi$ to a function on the homogeneous space $\left(\operatorname{Spin}(4) / S U(2) \simeq S^{3}\right)^{4}$, and can be obtained explicitly by group averaging $P_{h} \varphi\left(g_{1}, B_{1} ; \ldots ; g_{4}, B_{4}\right)=$ $\int \mathrm{d} h_{1} \ldots \mathrm{d} h_{4} \varphi\left(g_{1} h_{1}, B_{1} ; \ldots ; g_{4} h_{4}, B_{4}\right)$.

- The kinetic operator is given by

$$
\mathcal{K}\left(g_{i}, B_{i}\right)=\prod_{i=1}^{4}\left(B_{i}^{2}+\square_{G_{i}}-\frac{m^{2}}{4}\right)
$$

with $\square_{G_{i}}$ being the Laplacian operator on the $i$ th copy of the group manifold $G=\operatorname{Spin}(4)$ or on the homogeneous space $S^{3}$, the square $|B|^{2}$ (or $\left|b_{i}^{+}\right|^{2}$ ) is taken using the fundamental Killing form on the Lie algebra, and $\mathrm{m}^{2}$ is an arbitrary positive constant.

- The projector $P_{g}$ imposes invariance under the diagonal action of the group Spin(4) on the group and Lie algebra variables: $P_{g} \varphi\left(g_{1}, B_{1} ; \ldots ; g_{4}, B_{4}\right)=$ $\int \mathrm{d} g \varphi\left(g_{1} g, g^{-1} B_{1} g ; \ldots ; g_{4} g, g^{-1} B_{4} g\right)$.

- The vertex or interaction operator $\mathcal{V}$ is given by

$$
\mathcal{V}\left(g_{i j}, B_{i j}\right)=\prod_{i \neq j=1}^{5} \delta\left(g_{i j} g_{j i}^{-1}\right) \delta\left(B_{i j}-B_{j i}\right)
$$

The four variables appearing in each field, interpreted as being associated with the four triangles in the boundary of the tetrahedron $i$ corresponding to it, are represented in figure 2.

Let us now explain motivation and geometric content of all these ingredients (for more details, see [21]).

The key to the geometric interpretation of the various ingredients and of the whole model is the geometric interpretation of the field $\varphi\left(g_{i}, B_{i}\right)$ itself. We can think of it as the second quantization of a tetrahedron whose geometry is characterized by the four pairs of first-order variables $g_{i}$ representing elementary parallel transports of a Lorentz connection along paths dual to the triangles of the tetrahedron, and $B_{i}$ representing Lie algebra variables (or bivectors) associated with the same triangles [2, 3]. In addition, we assume that a reference frame is 


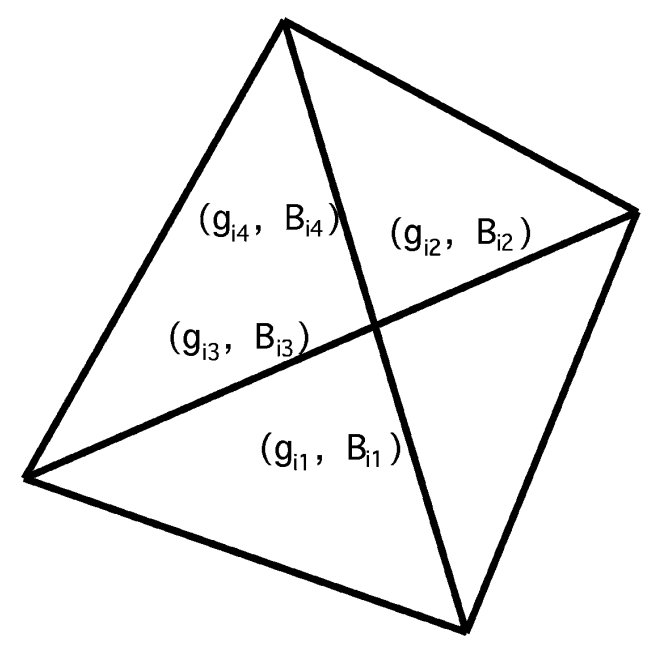

Figure 2. The variables associated with the four triangles of each tetrahedron, a group element $g$ and a Lie algebra element $B$.

associated with a tetrahedron and, thus, to each field $\varphi$, and that the $B$ variables are expressed in this frame.

Let us now look at the geometric content of the interaction term. First of all, as in other GFT models, the combinatorics of field arguments is chosen to respect the combinatorics of triangles in a 4-simplex. It is this feature that characterizes GFTs as peculiarly non-local field theories. Second, the symmetry imposed by the projector $P_{g}$ amounts to the requirement that the reference frame associated with each tetrahedron should be arbitrary. In other words, one should be free to relate this frame by means of an arbitrary parallel transport to some given, and equally arbitrary, frame associated with the 4-simplex as a whole. This parallel transport is effected by the group element $g$ integrated over in the projection $P_{g}$, that can be thought of as being associated with (half) dual edges in the 2-complex dual to the triangulation to which the 4-simplex belongs. One can do the same for all the tetrahedra/fields in the vertex term. This means that their different variables all come from a single set of triangle variables for the 4-simplex they belong to, which are then parallel transported to different locations (the boundary tetrahedra) in the simplex. This symmetry imposition amounts to a relaxation of the usual symmetry imposed on the group variables alone in the usual GFTs (and interpreted as imposing the closure constraint of BF-like theories). The net result is that the field is not invariant anymore under the diagonal action of the group, when seen as a function of the group elements only, but it becomes covariant, and this translates in representation space as a covariance, as opposed to invariance, of the tensors between the four representations associated with the triangles of the tetrahedron. The need to relax this invariance to a covariance requirement has also been emphasized, from a canonical perspective, by Alexandrov [30]. By a change of variables one can move the group variables defining the projection operators $P_{g}$ into the vertex function $\mathcal{V}$, which are then defined on non-projected fields $\phi$ 's $($ or $\varphi$ ). The result is a vertex function given by

$\mathcal{V}\left(g_{i j}, B_{i j}\right)=\int \prod_{i=1}^{5} \mathrm{~d} g_{i} \prod_{i \neq j=1}^{5} \delta\left(g_{i j} g_{i} g_{j}^{-1} g_{j i}^{-1}\right) \prod_{i \neq j=1}^{5} \delta\left(B_{i j}-g_{i}^{-1} g_{j} B_{j i}\left(g_{i}^{-1} g_{j}\right)^{-1}\right)$.

The geometric meaning is now transparent. The delta functions on the group impose that the parallel transport $g_{i j} g_{i} g_{j}^{-1} g_{j i}^{-1}$ of the Lorentz connection along the boundary of the wedge 


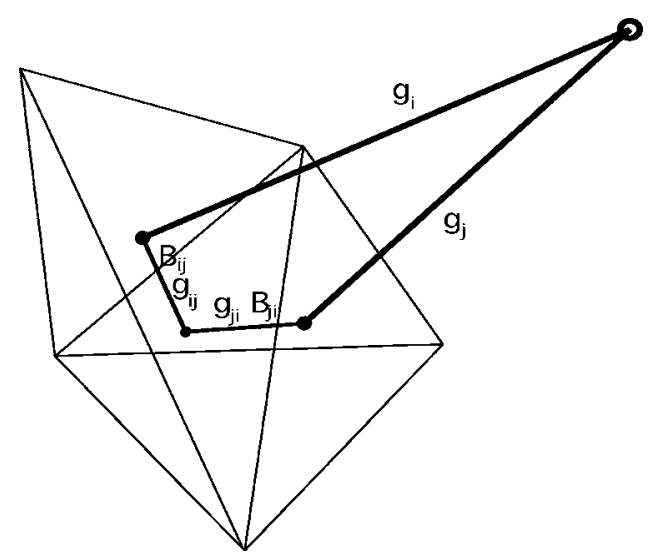

Figure 3. The variables associated with each wedge in the (dual of the) simplicial complex: two group elements $g_{i}$ and $g_{j}$ representing parallel transports from the center of the 4-simplex to the center of the two tetrahedra $i$ and $j$ sharing the triangle $i j$ dual to the wedge; two group elements $g_{i j}$ and $g_{j i}$ (arguments of two fields corresponding to the tetrahedra sharing the same triangle) representing parallel transports from the center of the two tetrahedra to the center of the triangle; the two Lie algebra variables $B_{i j}$ and $B_{j i}$ representing the $B$ variable associated with the triangle $i j$ seen in the reference frame of the tetrahedron $i$ and of the tetrahedron $j$, respectively.

(portion of the dual face inside each 4-simplex) associated with the triangle $i j$ shared by the tetrahedra labeled $i$ and $j$ and living inside the 4-simplex that contains both these tetrahedra is flat. This is consistent with our piecewise-flat context. The deltas on the Lie algebra impose that the Lie algebra variables (discrete $B$ field) associated with the same triangle in two different tetrahedra are identified only after parallel transport from the center of one tetrahedron (where they are originally defined) to the center of the 4-simplex, i.e. to the single reference frame in which they are in fact the same, and back to the other tetrahedron, with this parallel transport affected by the group elements $g_{i}{ }^{5}$.

The variables associated with each wedge are shown in figure 3.

We see therefore that the interaction term of the GFT model enforces a symmetry requirement (local Lorentz invariance) and the trivial kinematical geometry dictated by a piecewise-flat setting. Note that, because of the projectors $P_{B}$ and $P_{h}$ acting on the field $\varphi$, the variables $B_{i j}$ entering the vertex term will be constrained to be simple, and we will also have $g_{i j} \in S^{3}$.

Let us then give a look at the kinetic term. This is given by the product of four KleinGordon operators acting on the group manifold $G$ with a variable mass term given by $B^{2}$ plus an arbitrary constant mass square shift, one for each triangle in the tetrahedron the field refers to. This choice [16], first of all, relaxes the identification between the discrete bivector $B$ associated with the triangle and the Lie algebra generator $J$ seen as an operator acting on functions of the group, and thus between its modulus square and the (first) Casimir of the algebra, which in turn is defined up to an arbitrary constant shift. This specific choice is the simplest Lorentz invariant one achieving the above. Obviously, others could be considered. On the one hand, in the present context, this step allows us to deal with both $B$ and $g$ variables at the same time, it leads to a good simplicial path integral, as we are going to see, and keeps the simplicial geometry of the model fully manifest. On the other hand, we may suspect

5 This piecewise flat geometric requirements have also been nicely discussed in [24, 25], even though they do not seem to be fully implemented in the quantization and thus in the model presented there. 
already at this stage that it will lead us away from the dynamics of BF theory, even in the absence of simplicity constraints, since the conjugate nature of these variables (characteristic of BF theory) is then not imposed in our model.

The additional projectors $P_{h}$ led to a Klein-Gordon operator on the homogeneous space $S^{3}$. It results in the amplitudes depending on a single-angle parameter on the group manifold. It is mainly motivated by the specific discrete gravity action this leads to. However, it also makes the same amplitudes, when expanded in representations of the Lorentz group, involve only class 1 representations, as in the Barrett-Crane model and in the other new spin foam models $[22,24,25]$. We know that this represents partial imposition of the simplicity constraints in terms of the connection variables, following their (partial) imposition on the Lie algebra generators identified with the discrete $B$ variables in a geometric quantization of BF theory. Here, on the other hand, the same constraints are fully implemented on the Lie algebra variables $B$. Therefore, we could expect this additional restriction to be redundant, to some extent. We will discuss later on to what extent this is in fact true.

The simplicity constraints on the $B$ variables are imposed by the factor $\mathcal{C}\left(B_{i}\right)$ that is inserted for each field and thus for each tetrahedron in the triangulation, separately in each 4-simplex to which the tetrahedron belongs. In fact, the GFT kinetic term defines the gluing of two 4-simplices in the 4D triangulation associated with each Feynman diagram (see $[2,3]$ ) across a common tetrahedron, represented by the two fields in the GFT kinetic term (the same tetrahedron seen in two different 4-simplices). The simplicity constraints amount to the imposition that the four bivectors associated with the four triangles $f$ of the tetrahedron $t$ all belong to the same hypersurface (in flat space), i.e. they are all normal to the same unit 4-vector, interpreted as the normal to the tetrahedron [22, 24-26, 30]: $\exists N_{t} \in S^{3} / B_{f}^{I J} n_{t J}=0 \forall f \subset t$. In this case, the bivectors are interpreted as defining the so-called 'area bivectors' $A_{f}=e_{1} \wedge e_{2}$ formed by the wedge product of two edge (tetrad) vectors $e_{1,2}$ of the triangle $f$. A dual version of the same constraints is the requirement that the four bivectors are such that their duals are normal to the same unit normal vector and thus belong to the same hypersurface: $\exists N_{t} \in S^{3} /\left(* B_{f}\right)^{I J} N_{t J}=0 \forall f \subset t$. In this case, they are interpreted as dual to the same area bivectors: $B_{f}=* A_{f}{ }^{6}$. To the above constraints, it is necessary to add the so-called closure constraint, imposing that the four bivectors associated with the triangles in a given tetrahedron sum to zero, i.e. that the tetrahedron 'closes'. Only when all these conditions are satisfied it is possible to invert the set of bivectors associated with the triangles of the simplicial complex for a set of tetrad vectors associated with the edges of the same and specifying a simplicial geometry. It is possible to show, moreover, that these sets of constraints represent the discrete version of the continuum Plebanski constraints. The constraining factor $\mathcal{C}\left(B_{i}\right)$, brought into the action by the mentioned map $P_{B}$, has thus the form

$$
\begin{aligned}
\mathcal{C}\left(B_{i}\right) & =\int_{S^{3} \simeq S U(2)} \mathrm{d} N_{t} \prod_{i=1}^{4} \delta^{(3)}\left(b_{i}^{-} \mp N_{t} \triangleright b_{i}^{+}\right) \delta\left(\sum_{i} b_{i}^{+}\right) \\
& =\int_{S^{3} \simeq S U(2)} \mathrm{d} N_{t} \prod_{i=1}^{4} \delta^{(3)}\left(b_{i}^{-} \mp N_{t} b_{i}^{+} N_{t}^{-1}\right) \delta\left(\sum_{i} b_{i}^{+}\right) \\
& =\int_{S^{3} \simeq S U(2)} \mathrm{d} N_{t} \prod_{i=1}^{4} \int_{\mathfrak{s u}(2)} \mathrm{d} b_{i} \delta\left(B_{i}-\left(b_{i}, \pm N_{t} b_{i} N_{t}^{-1}\right)\right) \delta\left(\sum_{i} b_{i}^{+}\right),
\end{aligned}
$$

6 Note that in the absence of the Immirzi parameter, the two new spin foam models [22, 24-26], based on imposing the simplicity constraints on coherent states of the Lorentz group, are distinguished exactly by the above choice. 
where we have written the simplicity constraints using the self-dual/anti-self-dual decomposition of the Lie algebra elements $B_{i}$ 's, and the identification of elements of the homogeneous space $S^{3}$ with the Spin(4) elements mapping the reference unit vector $(1,0,0,0)$ into them, and further parametrized them as $S U(2) \times S U(2)$ elements as $N_{t}=\left(N_{t}^{+}, N_{t}^{-}\right)=\left(1, N_{t}\right)$ at the cost of a slight abuse of notation (see [22]). The two possible signs $\mp$ correspond to the first (second) way of imposing the simplicity constraints, respectively. The arbitrariness of the normal vector $N_{t}$ is enforced by the integration over $S^{3}$. In the last line, we have defined the simple bivectors $b_{i}=\left(b_{i}, \pm b_{i}\right)$ in self-dual/anti-self-dual decomposition, with similar abuse of notation, and with the same sign ambiguity.

Note that, due to the delta functions imposing the closure constraint, a (straightforward) regularization of the action and, later, of the amplitudes of the model will be needed.

Note also that the anti-self-dual variables $b_{i}^{-}$of the field $\varphi$ are not coupled among different fields, but are instead fixed completely as functions of the $b_{i}^{+}$by the map $P_{B}$ (simplicity constraints) in each field separately.

\subsection{Feynman amplitudes: discrete gravity path integrals}

The quantization of the model is obtained by the partition function defined as a perturbative expansion in Feynman diagrams in the coupling constant $\lambda$ :

$$
Z=\int \mathcal{D} \phi \mathrm{e}^{\mathrm{i} S[\phi]}=\sum_{\Gamma} \frac{\lambda^{V_{\Gamma}}}{\operatorname{sym}(\Gamma)} Z_{\Gamma},
$$

where $V_{\Gamma}$ is the number of vertices in the Feynman diagram $\Gamma$ (see $\left.[2,3]\right), \operatorname{sym}(\Gamma)$ is the order of automorphisms of the diagram/complex, and $Z_{\Gamma}$ is the corresponding Feynman amplitude, whose construction is detailed in [16], for this general class of models, and in [21] for this specific one and its 3D counterpart. The key ingredient is the non-trivial propagator, the inverse of the kinetic term 4. This being a product of Klein-Gordon operators on the homogeneous space $S^{3} \simeq \operatorname{Spin}(4) / S U(2)$, thanks to the projection operators $P_{h}$, its inverse is taken to be the product of the Feynman propagator on the same homogeneous space, in turn equal to the propagators on the group $S U(2)$, due to the isomorphism between the two spaces, with variable mass given by $b_{i}^{2}-\frac{m^{2}}{4}$. From now on we set $m^{2}=1$ because this simplifies the resulting formulae. They can also be written in terms of the analogous propagators on the full Spin(4) group acted upon by the same projectors $P_{h}$ (see [31]). The resulting schematic form of the amplitudes is

$Z_{\Gamma}=\prod_{(e v)} \int_{\operatorname{Spin}(4)} \mathrm{d} g_{v e} \prod_{(e f)} \int_{\mathfrak{s u}(2)} \mathrm{d} b_{e f}^{+} \int_{\mathfrak{s u}(2)} \mathrm{d} b_{e f}^{-} \mathrm{d} \tilde{b}_{e f}^{-} \prod_{(e v)} \mathcal{C}\left(B_{f \subset e ; v}\right) \prod_{f} A\left[B_{e f}, g_{v e}\right]$.

The amplitudes factorize per dual face, apart from the measure factor $\mathcal{C}$ imposing the simplicity constraints, which is associated with each dual edge $e$ (tetrahedron) for each vertex $v$ (4simplex) it touches (belongs to). They depend on one $\mathfrak{s u}(2)$ Lie algebra variable $b_{e f}^{+}$for each tetrahedron $e$ sharing the triangle dual to the face $f$, two similar (anti-self-dual) Lie algebra elements associated with the same tetrahedron-triangle pair, one for each 4-simplex $v$ sharing it, and one group element $g_{e v}=\left(g_{v e}\right)^{-1}$ for each half-dual edge incident to a given vertex $v$. Recall that all the anti-self-dual Lie algebra elements are fully fixed as functions of the self-dual ones, by the constraints $C$. Equivalently, one can think of a single $\mathfrak{s o ( 4 ) ~ L i e ~ a l g e b r a ~}$ element $B_{e f ; v}=\left(b_{e f}^{+}, b_{e f}^{-}\right)_{v}$ associated with each dual face (triangle) in each tetrahedron sharing it, within each one of the two 4-simplices $v$ to which the tetrahedron belongs, and with only the self-dual components of these Lie algebra elements being identified from one tetrahedron to the next and across 4-simplices. The full holonomy around the dual face is 
given by $H=\prod_{e \in \partial f} g_{e}$ (having chosen an arbitrary ordering of the edges in the boundary of the face), where we have defined $g_{e}=g_{v e} g_{e v^{\prime}}$. They have the generic form

$$
A\left[B_{e f}, g_{e v}\right]=\mu\left(H_{f}, B_{e f}\right) \mathcal{W}\left(g_{e v}, B_{e f}\right) \mathrm{e}^{\mathrm{i} S_{R}^{f}\left[B_{e f}, H_{f}\right]} \mathrm{e}^{\mathrm{i} S_{c}^{f}\left[B_{e f}, H_{f}\right]} .
$$

We now discuss the various contributions to these amplitudes.

The first term to note is the action term $S_{R}^{f}\left(B_{e f}, H_{f}\right)=\left|B_{e f}\right|\left|\left[\theta_{f}\left(H_{f}\right)\right]\right| .\left|B_{e f}\right|$ is the modulus of a Lie algebra variable associated with the dual face $f$, and thus to the corresponding triangle in $\Delta$, in the reference frame of one of the tetrahedra sharing it. $\theta_{f}\left(H_{f}\right)$ is the distance on the homogeneous space $S^{3}$ defined by the group element $H_{f}$, which computes the simplicial curvature associated with the dual triangle. The holonomy is defined starting from and ending to the same tetrahedron in whose frame we defined $B_{e f}$; the choice of the tetrahedron, and thus of the frame, is immaterial [24, 25]. The notation [] indicates that the amplitude depends only on the equivalence class of distance angles corresponding to the same group element $H_{f}$; this is a result of the periodic boundary conditions imposed on the propagators of the compact manifold $S^{3}$. It is realized in practice by defining this distance to be $\theta \pm 2 \pi n$, with $\theta \in[0,2 \pi]$ and $n \in \mathbb{N}$ identifying each of the possible geodesics in $S^{3}$ on which the distance is computed, and summing over $n$, thus adding the variable $n$ to the configuration variables on which the amplitudes depend (see [16, 31] for details). Taking into account the product over dual faces in (9), this gives an action term associated with the whole triangulation $\Delta$ dual to $\Gamma$ given by $S_{R}=\sum_{f} S_{R}^{f}\left(B_{f}, H_{f}\right)=\sum_{f}\left|B_{f} \|\left[\theta_{f}\left(H_{f}\right)\right]\right|$. This action term characterizes the Feynman amplitudes of the model as a simplicial gravity path integral. However, it is the presence of the terms $\mathcal{C}_{e}$ and $\mathcal{W}_{f}$ that fully characterizes the geometric content of this action and thus the dynamics of the theory.

The simplicity constraints $\mathcal{C}_{e v}\left(B_{f \subset e}\right)$, as discussed above, impose the geometric restriction that the set of $B$ variables associated with the triangles of $\Delta$ are either identified with or dual to the area bivectors for the same triangles [22, 24-26]. These constraints ensure that the set of $B_{f}$ variables can be put in bijective correspondence with a set of tetrad vectors $E_{l}^{I}$ associated with the links of the triangulation $\Delta$, and thus with a unique (up to local Lorentz transformations) simplicial geometry [28]. In practice, they impose that each $B$ variable is of the form $\left(b_{f}, \pm N_{e} b_{f} N_{e}^{-1}\right)$ for an arbitrary vector $N_{e}$ common to all the bivectors associated with the same tetrahedron $e$, and that tetrahedra close in the hypersurface orthogonal to $N_{e}$. It can be rephrased by saying that the Lie algebra element $B_{f}$ is, up to the common Lorentz rotation $N_{e}$, the generator of a $U(1)$ subgroup of the diagonal $S U$ (2) or of a corresponding antidiagonal one, depending on the sign chosen. The vector $N_{e}$ can be set to the form $(1,0,0,0)$ by an appropriate gauge choice in each tetrahedron (see [22, 24, 25]).

The contributions $\mathcal{W}\left(g_{v e}, B_{e f}\right)$ are delta functions on the Lie algebra. Choosing an ordering $1, \ldots, N$ of the $N$ edges in the boundary of the dual face, fixing an arbitrary edge as the reference one and labeling it by 1 (with $N+1 \equiv 1$ ), and defining $g_{e^{\prime} e}=g_{e^{\prime} v} g_{v e}$, they have the form

$\mathcal{W}=\prod_{\bar{e}=1}^{N} \delta\left(B_{\bar{e}}-g_{1 N} \ldots g_{\bar{e}+1 \bar{e}} \ldots g_{21} \triangleright B_{1}\right)=\delta\left(B_{N}-g_{21} \triangleright B_{1}\right) \ldots \delta\left(B_{1}-g_{1 N} \ldots g_{21} \triangleright B_{1}\right)$,

where we simplified the notation $B_{e f} \rightarrow B_{e}$ as we are dealing with a single dual face.

These impose the obvious geometric requirement that the Lie algebra variables appearing in the different tetrahedra but corresponding to the same triangle, being the same fundamental Lie algebra element just expressed in different reference frames, can be obtained all from a single arbitrary one (here $B_{1}$ ) by parallel transports of this across the various simplices sharing the same triangle. Recall that the variables $B_{e f}$ are integrated over in the discrete partition function. If this was a free integration, then one could simply use the above deltas to eliminate 
all the redundant $B$ variables, leaving in the end a single one per dual face. This is what happens in the $3 \mathrm{D}$ case [21]. However, the simplicity constraints $\mathcal{C}_{e v}$ impose restrictions on these integrals, and these restrictions, together with the above deltas, imply further restrictions on the parallel transport (connection) variables $g_{e v}$. We leave a complete analysis of these restrictions to further work, but it is clear that they impose a consistency or compatibility requirement between simplicity constraints and gauge invariance, thus parallel transport ${ }^{7}$.

A careful analysis of the constraints $\mathcal{W}$, and of their interplay with the simplicity constraints $C$, has been performed in [36], after a first version of the present work had appeared. The analysis confirms the above and goes much further, in that it shows that these compatibility conditions can be enough to solve completely the connection degrees of freedom in terms of the tetrad or bivector ones, and thus go from a first-order formulation to a second-order one, which is fully equivalent to ordinary Regge calculus at the classical level. Moreover, the restriction on the connection degrees of freedom is such that the projection $P_{h}$ we have imposed on our fields that leads to our quantum amplitudes to depend only on the $S^{3}$ component of the group elements, with the consequences we have discussed, becomes unnecessary. In fact, they would result in the component of the holonomy along $\left(b_{f}, b_{f}\right)$ to be trivialized altogether, obtaining the same form and geometric interpretation even when working with generic Spin(4) group elements. However, at the same time the results of [36] suggest that the correct way of imposing the simplicity constraints may differ slightly but crucially from the one we adopted here. Having imposed the simplicity constraints at the level of each field $\varphi$ independently, by means of the map $P_{B}$, we end up with two normal vectors $N_{e v} \in S^{3}$ associated with the same tetrahedron $e$ one for each 4-simplex $v$, which our model treats as completely independent from each other. This has one important consequence. The restriction on the connection implied by the requirement that the two normals to two tetrahedra sharing the same triangle (either within the same 4-simplex or in two adjacent ones), both lie in the plane orthogonal to the area bivector associated with that triangle [36], is missing in our formulation. This requirement is important for the complete inversion of the connection variables as a function of the bivector variables; thus, for the definition of geometric dihedral angles from them, and it is not clear, at this stage, how much of this inversion one can still perform given our weaker form of the constraints. Note also that this weaker form of the constraints is the same imposed in the Barrett-Crane model (within a different formalism), and possibly the origin of the problems faced by it (see also [35]). How the stronger form of constraints can be imposed in a GFT context is the subject of work in progress [40].

Of particular interest is the last of these deltas

$$
\delta\left(B_{1}-g_{1 N} \ldots g_{21} \triangleright B_{1}\right)=\delta\left(B_{1}-H_{f} \triangleright B_{1}\right) .
$$

This imposes on the face holonomy the condition $H_{f}=\mathrm{e}^{\mathrm{i} \theta_{1} \hat{B}_{e}+\mathrm{i} \theta_{2} * \hat{B}_{e}}$ for arbitrary angles $\theta_{1,2}$; in other words, it imposes that $H_{f}$ lives in the $U(1) \times U(1)$ Cartan subgroup of Spin(4) and, moreover, that this is aligned with the subgroup generated by the two commuting Lie algebra elements $\hat{B}_{e}=\frac{B_{e}}{\left|B_{e}\right|}$ and $* \hat{B}_{e}$.

The geometric content of this condition is revealed by taking the simplicity constraints into account as well. First of all, note that the presence of this delta function reduces the gauge invariance of the Feynman amplitude $Z_{\Gamma}$ from the invariance under $B_{e f} \rightarrow G B_{e f} G^{-1}$, $H_{f} \rightarrow \bar{G} H_{f} \bar{G}^{-1}$ for arbitrary $G, \bar{G}^{8}$, that could be deduced from the action term alone and the simplicity constraints (we will see that also the other terms $\mu$ and $S_{c}$ would allow for this large

7 Analogous compatibility conditions were noticed in [29].

8 This follows from the fact that each propagator contributing in building up the Feynman amplitude is invariant under the conjugate action of the group $G$ on the homogeneous space $S^{3}$ and that it depends on each $B$ only through its modulus. 
symmetry) to the smaller $B_{e f} \rightarrow G B_{e f} G^{-1}, H_{f} \rightarrow G H_{f} G^{-1}$ for the same group element $G$. This is indeed the symmetry of BF theory (see, for example, [24, 25]). Consider now the simplicity constraints with a negative $\operatorname{sign} B_{e f}=\left(b_{e f},-n_{e} b_{e f} n_{e}^{-1}\right)$ implying that $B_{e f}$ is an area bivector $A_{f}$. This can be rewritten as $B_{e f}=N_{e} \triangleright * b_{e f}$ for the group element $N_{e}=\left(1, n_{e}\right)$ with $n_{e} \in S U(2)$ [22] and $b_{e f}=\left(b_{e f}, b_{e f}\right)$ in the self-dual/anti-self-dual notation. This implies (simplifying again the notation) that $H_{f}=\mathrm{e}^{\mathrm{i} \theta_{1} \hat{B}_{f}+\mathrm{i} \theta_{2} * \hat{B}_{f}}=N_{e} \mathrm{e}^{\mathrm{i} \theta_{1} * \hat{b}_{f}+\mathrm{i} \theta_{2} \hat{b}_{f}} N_{e}^{-1}=N_{e} h_{f} N_{e}^{-1}$ with $h_{f}=\mathrm{e}^{\mathrm{i} \theta_{1} * \hat{b}_{f}+\mathrm{i} \theta_{2} \hat{b}_{f}}$. Let us then take into account the symmetry noticed above. The amplitudes, and in particular the action term, depend on $H_{f}$ and $B_{f}$ only up to their simultaneous rotation by an arbitrary group element $G$. Let us choose this as $G=N_{e}$. Then, it results that the amplitudes are the same as those computed from $b_{f}$ and $h_{f}$. More precisely, the action $S_{R}$ depends only on the modulus of $b_{f},\left|b_{f}\right|=\left|B_{f}\right|=\left|A_{f}\right|$ and on the distance of $S^{3}$ corresponding to the holonomy $h_{f}$. But $h_{f}=\mathrm{e}^{\mathrm{i} \theta_{1} * \hat{b}_{f}+\mathrm{i} \theta_{2} \hat{b}_{f}}$, with $b_{f}=\left(b_{f}, b_{f}\right)=A_{f}$, thus with $\mathrm{e}^{\mathrm{i} \theta_{2} \hat{b}_{f}}$ belonging to the diagonal $S U(2)$ subgroup of $\operatorname{Spin}(4)$. Therefore we conclude that the angle measuring the distance on $S^{3}$ and entering the action $S_{R}$ is the component of $h_{f}$ along $* b_{f}=* A_{f}$, or of $H_{f}$ along $B_{f}$, i.e. $\theta_{f}\left(h_{f}\right)= \pm \theta_{1}$. We can therefore interpret the contribution of each dual face to the simplicial action as $\left|B_{f}\right|\left|\left[\theta_{f}\right]\right|=\operatorname{tr}\left(B_{f} F_{f}\right)=\operatorname{tr}\left(B_{f} \ln H_{f}\right)$ (the trace is in the Lie algebra) with the additional restriction (that can be included in the measure of integration over the $B$ and $g$ variables by means of a simple Heaviside function) $\operatorname{tr}\left(B_{f} F_{f}\right)>0$. This same restriction has been argued for as a (pre-)causality condition needed to define causal transition amplitudes for BF theory and gravity in [19]. The simplicity constraints imply that each $B$ variable comes from an appropriate set of discrete tetrad variables. In turn, this implies that the simplicial action $S_{R}$ can be understood schematically as $S_{R}(E, g)=\sum_{f} \operatorname{tr}\left(* A_{f}(E) F_{f}(g)\right)$, i.e. as a simplicial action for 4D gravity of a firstorder Regge type [16, 23, 27], in turn a discrete version of the Palatini action for 4D gravity. Equivalently, this shows our model to be a simplicial gravity path integral for a discrete Plebanski formulation of 4D gravity as a constrained BF theory.

Finally, let us mention the last two contributions to the Feynman amplitudes $Z_{\Gamma}$. The first is a measure term $\mu\left(B_{e f}, H_{f}\right)$ which is a real function of $\left|B_{f}\right|$ and $H_{f}$ only (with parametric dependence on the number of $N$ dual edges/vertices in the dual face $f$ ). The second is an additional contribution $S_{c}$ to the classical action $S_{R}$ that we can interpret as a quantum correction to the same. It has the same dependence on the basic variables as $\mu(B, H)$. These two terms are identified [16] as the modulus and phase, respectively, of the complex function:

$$
\begin{aligned}
v(H, B, N) \propto & \frac{-\mathrm{i}}{(N-1) !} \frac{1}{\sin \left(\theta\left(H_{f}\right)\right)}\left(\frac{\left|\left[\theta\left(H_{f}\right)\right]\right|}{\left|B_{f}\right|}\right)^{N-1} \\
& \times \sum_{K=0}^{N-2} \frac{(N+K-2) !}{K !(N-K-2) !}\left(\frac{\mathrm{i}}{2\left|B_{f}\right|\left|\left[\theta\left(H_{f}\right)\right]\right|}\right)^{K} .
\end{aligned}
$$

Their explicit expressions and asymptotic form for $\left|B_{f}\right| \rightarrow \infty, H_{f} \neq I$ are reported and discussed in $[16,21]$. This analysis, which is the analog of the semi-classical expansion $j \rightarrow \infty$ performed in usual spin foam models, is easily done for arbitrary triangulation, thanks to the fact that the full amplitude, modulo the various constraints (whose form is of course unmodified in the asymptotic regime), i.e. $v(B, H, N) \mathrm{e}^{\mathrm{i} S_{R}(B, H)}$, is given by an Hankel function, whose asymptotic formulae can then be used. The end result is that indeed the terms $S_{c}$ give subdominant large-scale corrections (of $1 / R^{n}$ type) in this regime to the dominant Regge term. 
In the end, the aim of obtaining a GFT model reproducing the Plebanski formulation of gravity (1) in a simplicial setting, and thus (implicitly ${ }^{9}$ ) a new promising spin foam model for $4 \mathrm{D}$ quantum gravity is realized.

\section{Discussion}

\subsection{Features of the model}

In the 3D case, and in 4D in the absence of the simplicity constraints, the static-ultralocal truncation of the generalized GFT models reproduces the usual 3D gravity spin foam model, i.e. the Ponzano-Regge model, and the analogous spin foam model for 4D BF theory $[16,21]$. This amounts to maintaining the same field variables, symmetries and interaction, but dropping the derivative (and variable mass) terms in the kinetic term, being left with simple delta functions. One can consider here a similar truncation and analyze the corresponding Feynman amplitudes.

Because of the projections $P_{h}$, and because of the constraints on the connection following the imposition of simplicity on the bivectors $B$, that we discuss below, the amplitudes will not force the dual face holonomies to be flat, as in BF. The simplicity constraints $\mathcal{C}(B)$ will still restrict the Lie algebra elements to be simple, and, in conjunction with the symmetry projectors $P_{g}$ will still imply further restrictions $\mathcal{W}$ on the connection variables ensuring compatibility between simplicity conditions and parallel transport. Because of the projections $P_{h}$ the resulting model can be expected to be similar to the Barrett-Crane spin foam model, also due to the weak form of the simplicity constraints we have chosen. However, because of the mentioned compatibility restrictions, thus because of the modified symmetry requirement $P_{g}$, we may still obtain a different model. In particular, we expect a stronger correlations among 4-simplices, affected by the matching of $B$ variables (and not only their modulus) across them, even if the connection variables would turn out to be not sufficiently constrained to be geometric.

The compatibility conditions $\mathcal{W}(B, g)$ are another aspect of the new model that need further study. First of all, one needs to clarify which restrictions they impose, exactly, on connection degrees of freedom, as we have discussed. Most important, maybe, is a clearer understanding of their geometric meaning and of their interpretation from the point of view of the canonical quantization of Plebanski action. One could in fact conjecture a relation between these constraints and the secondary second-class constraints identified in [32] and whose importance for the spin foam quantization has been emphasized in [30]. In particular, in [30] it is argued that the presence of these secondary second-class constraints is a necessary consequence of the simplicity conditions when considered together with the relaxed Lorentz covariance of the intertwiners. Moreover, [30] argues that these constraints would be essential for a proper integration over the connection variables by modifying the path integral measure with respect to the one in BF theory. Both these origin, features and consequences are shared by our constraints $\mathcal{W}(B, g)$, thus supporting our conjecture. In fact, they are (at least in the stronger form of [36]) equivalent in terms of resulting restrictions on the connection to the discrete gluing conditions ('edge simplicity') identified and studied in [38].

The quantum corrections $S_{c}$ to the classical Regge action $S_{R}$ deserve more study as well. Three features can be already noticed. First of all, they follow directly from the choice of the GFT action that therefore somehow fixes not only which terms will appear in the large

\footnotetext{
9 The re-writing of our quantum amplitudes in the pure spin foam form, i.e. as a function of group representations only, can be straightforwardly obtained. This has been done for the simpler but analogous models in [16]. While such re-writing can be useful, it does not modify the content of the model.
} 
distance limit or in any other approximate regime of the amplitudes but also their relative coefficients. This is also true for the corrections appearing in the $j \rightarrow \infty, H_{f} \approx I d$ limit of the simplicial action defined from coherent states in [28]. Second, this approximation would be the analog of the approximation $\left|B_{f}\right| \rightarrow \infty, H_{f} \approx I d$ of our full action $S_{R}+S_{c}$. In order to analyze this regime, however, a more careful study than the one in [16] that effectively applies to the regime $\left|B_{f}\right| \theta_{f}\left(H_{f}\right) \gg 1$ only is needed. Third, it can be shown [21] that these corrections originate directly from the gluing of 4-simplices, i.e. from the off-shell propagation of $B$ and $g$ variables across them. In fact, the amplitude for a single 4-simplex with boundary has a similar simplicial path integral form, depends exactly on the variables characterizing $\mathrm{BF} / \mathrm{Plebanski}$ theory on a manifold with boundary, with fixed-B boundary conditions, and involves the action $S_{R}$ only [21]. More generally, it is not clear, at this stage of development, whether the presence of the quantum corrections $S_{c}$, and in general the precise form of the measure $v$ is to be considered correct from the geometric point of view. It is possible that the GFT model itself will have to be amended in order to eliminate the corrections $S_{c}$ altogether and thus simplify the measure $v$.

An interesting extension of the model would instead be the one including the Immirzi parameter in the quantum amplitudes, and thus giving a quantization of (the simplicial version of) the Holst action. This parameter, crucial for the LQG formalism, enters prominently in the new spin foam models $[22,33]$, and affects significantly both their kinematics and dynamics. One would expect the Immirzi parameter to be straightforwardly introduced by modifying the simplicity map $P_{B}$ only. This generalized map involving the Immirzi parameter is easy to introduce, indeed, but this modification does not seem to be enough to achieve the expected form of the action in our simplicial path integral. The reason seems to be that a modification of the map $P_{h}$ seems needed as well, and in turn this can be understood as due to our weaker imposition of the simplicity constraints. We stress again that, indeed, with a stronger imposition [36] of the same, the maps $P_{h}$ could be avoided altogether and the connection would be completely fixed as a function of the $B$ 's. What is less clear at this stage is what should substitute the projections $P_{h}$ or, in other words, what restrictions on the connection degrees of freedom does the inclusion of the Immirzi parameter imply, given our weaker constraints. This is actually rather unclear also in the usual spin foam models [22, 33, 34].

While all of the above concerns the analysis or improvement of our model within the same generalized GFT formalism, an altogether different line of development is suggested by several of its features.

This new direction $[39,40]$ stems from the idea that, instead of relaxing the conjugate nature (at the classical level) of $B$ and $g$ variables in the definition of the model, treating them on equal footing as arguments of the GFT field, as we do here, one could instead take this conjugacy relation as the starting point for introducing the $B$ variables in the GFT formalism. This would mean to map the usual group field theories in which the field is a function of group elements into non-commutative and non-local field theories on several copies of the corresponding Lie algebra, using a generalized (non-commutative) Fourier transform, of the type developed in [41]. A first motivation for doing so is that, in the model we have presented, we are dealing with the Lie algebra of $\mathfrak{s o ( 4 )}$ (and its subalgebras) as an ordinary vector space, thus neglecting its non-commutative nature; this would lead to suspect that, in doing so, we are neglecting some relevant information that should instead be incorporated in a correct model using the $B$ variables as arguments of the GFT field. More precisely, the non-commutative nature of the algebra would result in a non-trivial star product for functions on it, when seen as functions on $\mathbb{R}^{3}$ (for $\mathfrak{s u}(2)$ ) or $\mathbb{R}^{6}$ (for $\mathfrak{s o}(4)$ ), as we do here. Moreover, the use of a non-trivial star product for the products of fields in the GFT action would result in a different composition rule for vertex amplitudes with propagators in the construction of the Feynman amplitudes of 
the model, and we have already noticed above how the rather puzzling quantum corrections $S_{c}$ to the BF action appearing in the model we have presented are the result of the way our individual vertex (4-simplex) amplitudes (itself given by a simple BF action) compose. Also, note that the maps $P_{B}$ could be turned into true projections, in a non-commutative setting, and one could avoid any divergence resulting from them, in particular from the use of an ordinary delta function to impose the closure constraint; this would be due to the fact that the non-commutative delta function on the Lie algebra [41] is a perfectly regular function when seen as a function on $\mathbb{R}^{3}$ (for $\mathfrak{s u}(2)$ ) or $\mathbb{R}^{6}$ (for $\mathfrak{s o}(4)$ ). The step to a non-commutative setting could then be motivated from the point of view of regularization only, even in the absence of the other mentioned motivations. Finally, we mention another hint for the need to move to a non-commutative setting, arising from the analysis of the model we have presented. It has recently been shown, both in 3D and in 4D [42], that effective scalar field theories on flat non-commutative spaces emerge naturally from group field theories, with the group manifold underlying the GFT providing the momentum degrees of freedom of the effective matter field, while the non-commutative position variables are expected to be related to the conjugate $B$ variables. A similar analysis performed using the generalized GFT formalism we have presented in this paper shows [43] that, while everything works fine even for these generalized GFT models as long as one focuses on the group sector, the expected matter field theory fails to emerge if one focuses instead on the Lie algebra sector of the same GFT models. This failure can be indeed traced back to having neglected the non-commutative structure of the same Lie algebra at the level of the fundamental GFT, i.e. in the definition of the generalized formalism itself.

At the same time, we think we should expect several features of the model we have presented to show up also in any new model using the $B$ variables and constructed by means of non-commutative tools. For example, the way the simplicial geometry is implemented at the level of the action and in particular the way the simplicity constraints are implemented, the interplay between simplicity constraints and gauge invariance, and the consequent compatibility relations encoded in the contributions $\mathcal{W}$ to the Feynman amplitudes.

\subsection{The dynamical triangulations sector}

Finally, let us conclude by showing a direct link between our new GFT model and the dynamical triangulations approach. This is made possible by the manifest simplicial geometric meaning of variables and amplitudes, that we can now put to use. We do not consider any additional causality restriction that one could impose on the model in order to obtain a restricted class of Feynman diagrams/triangulations summed over. These restrictions are crucial for the dynamical triangulations approach in its most modern form, called indeed causal dynamical triangulations [14]. Unfortunately, a field theoretic understanding of them in 4D in terms of some generalization of matrix models, and thus at the GFT level, is lacking, having been only recently achieved in the 2D case [44]. Consequently, we can make a link only with the 'old' dynamical triangulations approach: we obtain a model defined as a sum over equilateral triangulations (of arbitrary topology) weighted by a simplicial gravity action (Regge action plus corrections). The 4D DT approach is based on a second-order formulation of gravity where both area of triangles and dihedral angles between $(d-1)$-simplices are functions of fixed edge lengths. Our GFT model (4) corresponds to a first-order formulation where areas are given by $\left|B_{f}\right|$ and dihedral angles are encoded in the connection variables $g_{e}$. Therefore, in order to reproduce a DT setting we have to (a) kill all connection degrees of freedom, in turn fixing the local Lorentz invariance, and do so in such a way as to obtain dihedral angles in each 4-simplex corresponding to the values $\phi$ obtained from the edge lengths in the equilateral 
case; (b) kill 'direction' degrees of freedom in the $B$ 's and fix their modulus to a constant $\left|B_{f}\right|$ equal to the value of the area of triangle $f$ again in the equilateral case. Note that clearly this means having taken already into account, in both the $B$ and $g$ variables, the simplicity constraints. A candidate model for a GFT formulation of dynamical triangulations ${ }^{10}$ (in the Riemannian case), or for the DT subsector of our GFT model, is

$$
S=\frac{1}{2} \int \mathcal{D} g \phi\left(g_{i}\right) \mathcal{K}_{|B|}(g) \phi\left(g_{i}\right)+\frac{\lambda}{5 !} \int \mathcal{D} g \phi\left(g_{1 j}\right) \ldots \phi\left(g_{5 j}\right) \mathcal{V}_{h}(g)
$$

for $\mathcal{K}_{|B|}\left(g_{i}\right)=\prod_{i=1}^{4}\left(|B|^{2}+\square_{G_{i}}-\frac{1}{4}\right)$, with fixed $|B|$ and $\mathcal{V}_{h}(g)=\prod_{i \neq j=1}^{5} \delta\left(g_{i j} h g_{j i}^{-1}\right)$ with fixed $h=\mathrm{e}^{\mathrm{i} \phi} \in U(1)$. From this action, as it can easily be verified, one derives Feynman amplitudes of the same type of (9) without the constraining factors $\mathcal{C}$ and $\mathcal{W}$ nor any integration over the group or Lie algebra variables. The area of every triangle will be $|B|$ and the angle of holonomy will be $\theta_{f}=N_{f} \phi$, with $N_{f}$ being the number of 4-simplices sharing the triangle $f$. These parameters $|B|$ and $\phi$ can be of course chosen to match those computed from the edge lengths of an equilateral triangulation. The above model still defines a group field theory, but the group variables $g_{i}$ have only the effect of ensuring the gluing of simplices in the construction of the Feynman diagrams/amplitudes, and then disappear from the same (in the bulk, while they will still label boundary states whose kinematical variables, however, will not have any dynamics). One obtains therefore a sum over equilateral triangulations weighted by an amplitude involving the Regge action and depending on the above two parameters. When taken together with the weighting factor depending on the coupling constant $\lambda$ in (8), one notices that the GFT coupling constant can be interpreted as the exponential of (i times) the cosmological constant, that ends up multiplying the number of 4-simplices in the triangulation, matching exactly the usual DT construction. A Wick rotation can then be easily devised, as in the DT approach, to give a Euclideanized partition function, suitable for numerical analyses. The main differences from the usual dynamical triangulations approach are as follows: (a) the Regge term is augmented by the quantum corrections term $S_{c}$, a function of the same parameters, also resulting from (b) the measure term $v$ depending on the number of simplices per triangle. These terms have to be thoroughly analyzed, but it is a rather general result in the DT approach that its main features do not depend heavily on the details of the measure or of the action chosen, being rather dictated by entropic factors [14]. (c) The holonomy angle $\theta$ still enters the amplitudes through its equivalence class $\left[\theta_{f}\right]$ (in fact, the expression for the deficit angle in terms of the holonomy angle obtained from dihedral angles would be $\epsilon_{f}=2 \pi-\theta_{f}$ ). This is a direct consequence of our group-theoretic framework, with fields and propagators defined on a compact group manifold. This model will be analyzed elsewhere.

However, it already allows us to consider two scenarios, concerning the relation between GFTs and the dynamical triangulations approach. (I) The reduced GFT model matching the dynamical triangulations amplitudes may be still too general to achieve a good continuum limit. This would be the perspective coming from the dynamical triangulation side. In fact, first of all, it lacks the causality restrictions we mentioned above and that proved so crucial in recent developments: it still sums over different topologies and it does not incorporate any foliation structure for the triangulations of trivial topology. Second, it does include, in the configurations summed over, pseudo-manifolds, i.e. manifolds with conical singularities, whose characterizing features are also not understood at the field theoretic level. In this situation, with trivial amplitudes associated with the simplicial complexes, entropic considerations dominate and the model is likely too pathological.

${ }^{10}$ It is also possible that restriction to the dynamical triangulations sector of our full GFT model is achieved dynamically, without an explicit restriction of the initial model [16]. 
However, a second perspective is possible, coming from our GFT approach. (II) One could argue that the model is already too restricted, and that its pathologies are possibly cured within the full model instead. In particular, the presence of non-trivial amplitudes is crucial for both symmetry and renormalization considerations. The restricted model implies a trivialization of the gauge symmetries of the full one, with a consequent trivialization of the Ward identities, that lead to non-trivial relations among amplitudes associated with different Feynman diagrams (simplicial complexes). Complexes that would not be counted twice in the full sum, because of symmetries, are now distinguished in terms of their amplitudes depending on pure combinatorics. Moreover, the non-trivial amplitudes can be crucial in implementing a good renormalization procedure, and in devising suitable approximate regimes in which manifold-like configurations dominate with respect to pseudo-manifold ones. Recent results in $3 \mathrm{D}$ confirm this possibility [45].

\section{Conclusions}

We have presented a new GFT/spin foam model for 4D quantum gravity. It is based on a recent extension of the GFT formalism, in which the field depends on both group and Lie algebra variables, representing the discrete analog of the variables in a BF-like formulation of gravity. The new model allows a straightforward implementation of the simplicity constraints that give gravity from BF theory and has quantum amplitudes with the explicit form of simplicial path integrals for gravity. In doing so it sidesteps some ambiguous aspects of the usual spin foam quantization procedure. The geometric interpretation of the variables and of the different contributions to the quantum amplitudes is also made manifest. Moreover, thanks to the links with other discrete approaches to quantum gravity, that we have explicitly shown, its usefulness and interest may well extend beyond the spin foam or loop quantum gravity framework. While there are still several aspects of the new model that should be analyzed further, and interesting variations of the construction leading to it that can be considered, we believe that the new model represents an altogether new approach to the spin foam quantization of gravity and opens up a whole landscape of possible new developments, some of which we have discussed in some detail.

\section{Acknowledgments}

We thank T Tlas, with whom part of this line of research has been developed, for many useful discussions, criticisms and suggestions. Special thanks are due to V Bonzom and M Smerlak, and to S Alexandrov, for their interest, insightful criticisms and help. We also thank A Baratin, B Dittrich, J Engle, E Livine, K Noui and C Rovelli for comments on this work. Financial support from the A Von Humboldt Foundation through a Sofja Kovalevskaja Prize is gratefully acknowledged.

\section{References}

[1] Oriti D (ed) 2009 Approaches to Quantum Gravity (Cambridge: Cambridge University Press)

[2] Oriti D 2009 Approaches to Quantum Gravity ed D Oriti (Cambridge: Cambridge University Press) (arXiv:gr-qc/0607032)

[3] Oriti D 2007 Quantum Gravity ed B Fauser, J Tolksdorf and E Zeidler (Basel: Birkhaeuser) (arXiv: gr-qc/0512103)

[4] Freidel L 2005 Int. J. Phys. 44 1769-83 (arXiv:hep-th/0505016)

[5] David F 1985 Nucl. Phys. B 45257

Ginsparg P 1991 Matrix models of 2-d gravity arXiv:hep-th/9112013 
[6] Gross M 1992 Nucl. Phys. Proc. Suppl. 25A 144-9

[7] Ambjorn J, Durhuus B and Jonsson T 1991 Mod. Phys. Lett. A 6 1133-46

[8] Reisenberger M and Rovelli C 2001 Class. Quantum Grav. 18 121-40 (arXiv:gr-qc/0002095)

[9] Oriti D 2007 PoS arXiv:0710.3276

[10] Oriti D 2001 Rep. Prog. Phys. 641489 (arXiv:gr-qc/0106091)

Perez A 2003 Spin foam models for quantum gravity Class. Quantum Grav. 20 R43 (arXiv:gr-qc/0301113)

[11] Rovelli C 2006 Quantum Gravity (Cambridge: Cambridge University Press)

[12] Di Mane A, Livine E and Oriti D Second quantization of spin networks and a Fock space for LQG and group field theory (in preparation)

[13] Williams R 2009 Approaches to Quantum Gravity ed D Oriti (Cambridge: Cambridge University Press)

[14] Ambjorn J, Jurkiewicz J and Loll R 2005 Phys. Rev. D 72064014 (arXiv:hep-th/0505154) Ambjorn J, Jurkiewicz J and Loll R 2006 Contemp. Phys. 47 103-17 (arXiv:hep-th/0509010)

[15] Ooguri H 1992 Mod. Phys. Lett. A 7 2799-810 (arXiv:hep-th/9205090)

[16] Oriti D and Tlas T 2008 Class. Quantum Grav. 25085011 (arXiv:0710.2679)

[17] Livine E and Oriti D 2003 Nucl. Phys. B 663231 (arXiv:gr-qc/0210064)

[18] Oriti D 2005 Phys. Rev. Lett. 94111301 (arXiv:gr-qc/0410134)

[19] Oriti D and Tlas T 2006 Phys. Rev. D 74104021 (arXiv:gr-qc/0608116)

[20] Oriti D 2006 Phys. Rev. D 73061502 (arXiv:gr-qc/0512069)

[21] Oriti D and Tlas T 2010 Encoding simplicial geometry in group field theories Class. Quantum Grav. (arXiv:0912.1546 [gr-qc]) at press

[22] Freidel L and Krasnov K 2008 Class. Quantum Grav. 25125018 (arXiv:0708.1595)

[23] Caselle M, D’Adda A and Magnea L 1989 Phys. Lett. B 232457

Barrett J W 1994 Class. Quantum Grav. 11 2723-30 (arXiv:hep-th/9404124)

[24] Engle J, Pereira R and Rovelli C 2007 Phys. Rev. Lett. 99161301 (arXiv:0705.2388)

[25] Engle J, Pereira R and Rovelli C 2008 Nucl. Phys. B 798251 (arXiv:0708.1236)

[26] Livine E and Speziale S 2008 Europhys. Lett. 8150004 (arXiv:0708.1915)

[27] Conrady F and Freidel L 2008 Class. Quantum Grav. 25245010 (arXiv:0806.4640)

[28] Conrady F and Freidel L 2008 arXiv:0809.2280

[29] Bonzom V and Livine E 2008 arXiv:0812.3456

[30] Alexandrov S 2008 Phys. Rev. D 78044033 (arXiv:0802.3389)

[31] Camporesi R 1990 Phys. Rep. 1961

[32] Alexandrov S, Buffenoir E and Roche P 2007 Class. Quantum Grav. 242809 (arXiv:gr-qc/0612071) Buffenoir E, Henneaux M, Noui K and Roche P 2004 Class. Quantum Grav. 215203 (arXiv:gr-qc/0404041)

[33] Engle J, Livine E, Pereira R and Rovelli C 2008 Nucl. Phys. B 799136 (arXiv:0711.0146)

[34] Livine E and Oriti D 2002 Phys. Rev. D 65044025 (arXiv:gr-qc/0104043)

[35] Livine E and Oriti D 2007 J. High Energy Phys. JHEP02(2007)092 (arXiv:gr-qc/0512002)

[36] Bonzom V 2009 Class. Quantum Grav. 26155020 (arXiv:0903.0267)

[37] Bonzom V 2009 arXiv:0905.1501

[38] Dittrich B and Ryan J 2008 arXiv:0807.2806

[39] Baratin A and Oriti D 2010 The non-commutative representation for group field theories and simplicial gravity arXiv:1002.4723 [hep-th]

[40] Baratin A and Oriti D A new group field theory for 4d quantum gravity (in preparation)

[41] Freidel L and Majid S 2008 Class. Quantum Grav. 25045006 (arXiv:hep-th/0601004)

[42] Oriti D 2009 J. Phys. Conf. Ser. 174012047 (arXiv:0903.3970)

[43] Di Mare A and Oriti D 2010 Emergent non-commutative matter from a quantum geometric background Class. Quantum Grav. (arXiv:1001.2702 [gr-qc]) at press

[44] Benedetti D and Henson J 2008 arXiv:0812.4261

[45] Freidel L, Gurau R and Oriti D 2009 Phys. Rev. D 80044007 (arXiv:0905.3772) 